\title{
Angular anisotropy parameters for sequential two-photon double ionization of helium
}

\author{
I. A. Ivanov* and A. S. Kheifets \\ Research School of Physical Sciences and Engineering, The Australian National University, Canberra ACT 0200, Australia
}

(Received 5 November 2008; published 5 February 2009)

\begin{abstract}
We evaluate photoelectron angular anisotropy $\beta$ parameters for the process of sequential two-photon double electron ionization of helium within the lowest-order time-independent perturbation theory. Our results indicate that for photoelectron energies outside the interval $\left(E_{\text {slow }}, E_{\text {fast }}\right)$, where $E_{\text {slow }}=\omega-V_{\mathrm{He}^{+}}$and $E_{\text {fast }}=\omega-V_{\mathrm{He}}$, there is a considerable deviation from the dipole angular distribution, thus indicating the effect of electron correlation.
\end{abstract}

DOI: 10.1103/PhysRevA.79.023409

PACS number(s): $32.80 . \mathrm{Rm}, 32.80 . \mathrm{Fb}, 42.50 . \mathrm{Hz}$

\section{INTRODUCTION}

The rapid development of experimental techniques has given a considerable impetus to studies of multiphoton ionization in the xuv photon energy range. One of the most fundamental processes of this kind is two-photon double electron ionization (TPDI) of the helium atom. There are two physically distinguishable regimes of TPDI. One, the socalled nonsequential ionization (NSI) regime, occurs for photon energies exceeding the TPDI threshold at $39.5 \mathrm{eV}$. Another, the sequential ionization (SI) regime, takes over at photon energies above the ionization potential of the singly charged helium ion, $V_{\mathrm{He}^{+}}=54.4 \mathrm{eV}$. In this regime, the first photon knocks out one of the electrons from the neutral $\mathrm{He}$ atom. The second photon has enough energy to ionize the $\mathrm{He}^{+}$ion. According to this scenario, the two photoelectrons leave the $\mathrm{He}$ atom independently and are expected to have energies $E_{\mathrm{fast}}=\omega-V_{\mathrm{He}}$ and $E_{\text {slow }}=\omega-V_{\mathrm{He}^{+}}$, where $V_{\mathrm{He}}$ $=24.6 \mathrm{eV}$. Such an energy distribution is very different from the NSI regime where the single-differential, with respect to the energy, cross section (SDCS), is typically a broad concave curve [1].

The crossover from the NSI to the SI regime is not an abrupt one. The SI regime starts to manifest itself for photon energies even below the sequential threshold. It was shown in Ref. [2] that, when approaching the SI threshold from below, the SDCS exhibits rapid growth at the extremes of the energy sharing, which is a precursor of the two peaks appearing in the SDCS in the SI regime.

Because TPDI in the SI regime can take place without any interelectron interaction, the role of many-electron correlation in this process is expected to be insignificant. However, the mechanisms of TPDI become more subtle for very short laser pulses in the subfemtosecond regime. This was demonstrated in theoretical studies [3-6], which relied on numerical solution of the time-dependent Schrödinger equation (TDSE). This approach allowed one to reveal interesting effects related to the duration of the laser pulse. In principle, the SI and NSI regimes can be distinguished as follows. Under certain assumptions, the probability of SI scales as a square of the total duration of the pulse, while NSI scales only linearly [5]. These scaling laws are only valid if the

\footnotetext{
*Corresponding author: Igor.Ivanov@.anu.edu.au
}

populations of the initial atomic state and intermediate state of the $\mathrm{He}^{+}$ion do not change appreciably during the whole interval of time considered, which means that the laser pulse should be either short or of weak intensity [7]. It was found in Ref. [5] that for such pulses an additional (anomalous) component is present in the electron spectrum, which is due to sequential ionization and depends quadratically upon the pulse duration. This anomalous component was attributed to the process of relaxation. After the first ionization event takes place, the $\mathrm{He}^{+}$ion is in a nonstationary state. It relaxes subsequently to the ground state. During this relaxation, the photoelectrons still exchange energy. This leads to an increase of the SDCS in the region of the midenergy point $E_{S}=\left(E_{\text {fast }}+E_{\text {slow }}\right) / 2$.

This study was continued in work [6] where, in addition to the SDCS, photoelectron angular distributions were also studied. Authors used pulse parameters similar to those under investigation in Ref. [5]. It was found that, in agreement with the results of Ref. [5], correlation is responsible for the increase of the SDCS in the vicinity of the midenergy point (the "correlation valley" according to the authors' terminology). Moreover, the photoelectron angular distribution reveals quite distinctive features for different energy sharings. When ejected electrons share energy as $E_{\text {fast }}$ and $E_{\text {slow }}$, their angular distribution is strongly dipolar, which is a clear signature of an uncorrelated TPDI process. On the contrary, for the energies of the photoelectrons in the correlation valley, this distribution is dominated by a quadrupole contribution, thus indicating a very significant role played by the electron correlation.

Another manifestation of the effect of correlation in the TPDI SDCS for short pulses is the shift of the peaks towards each other [4-6]. These correlation effects are prevalent for short laser pulses. They are expected to disappear when the pulse duration becomes large. Nevertheless, it is a legitimate question to ask whether any traces of electron correlation remain in the photoelectron angular and energy distribution for long laser pulses. A useful tool for answering this question is the lowest-order, in respect to laser field, perturbation theory (LOPT), which allows one to study ionization processes driven by continuous electromagnetic radiation. LOPT was used in Ref. [8] to study the crossover from the NSI to SI regimes. In Ref. [2] an approach based on LOPT was used to compute the triply differential cross sections (TDCs) describing the angular distribution of the photoelectron pair in the coincident mode. 
It is known, however, that prescriptions of conventional LOPT lead to a problem in description of TPDI in the SI regime which manifests itself in the appearance of divergent terms in the SDCS $[2,8,9]$. The divergence problem can be avoided in LOPT based on amplitude equations taking into account depletion effects [7].

In the present paper, we use conventional LOPT to evaluate the anisotropy $\beta$ parameters characterizing the angular distribution of each individual photoelectron in a noncoincident mode. These parameters can be extracted unambiguously from a LOPT calculation, and their calculations do not suffer from the divergence problem. We shall also show that even in the limit of infinite pulse duration, there are some traces of electron correlation left in the angular distributions of the ejected electrons for extremely unequal energy sharings.

The paper is organized as follows. In the next section we give an outline of the theoretical procedure. Then we discuss the numerical results which we obtained for the SDCS and angular anisotropy parameters for several photon energies.

\section{THEORY}

\section{A. Implementation of LOPT}

We follow essentially the same theoretical approach which we used in Ref. [10] for calculation of the total integrated cross section (TICS) of TPDI in the NSI regime. We employ two versions of LOPT based on the length and Kramers-Henneberger $(\mathrm{KH})$ gauges of the electromagnetic interaction.

In the KH gauge, the operator of electromagnetic interaction takes the form

$$
\hat{H}_{\mathrm{int}}^{\mathrm{KH}}=\sum_{i=1}^{2}\left(\frac{Z}{r_{i}}-\frac{Z}{\left|\boldsymbol{r}_{i}+\hat{\boldsymbol{\alpha}}\right|}\right),
$$

where $\hat{\boldsymbol{\alpha}}=-\int_{0}^{t} \hat{\boldsymbol{A}}(\tau) d \tau, \hat{\boldsymbol{A}}$ is the quantized vector potential, and $Z=2$ for helium. For the operator 1 there are two lowestorder amplitudes connecting the initial and final states:

$$
M_{1}=\left\langle a, n+2\left|\hat{H}_{\mathrm{int}}^{\mathrm{KH}}\right| b, n\right\rangle
$$

and

$$
M_{2}=\oiint_{c} \frac{\left\langle a, n+2\left|\hat{H}_{\mathrm{int}}^{\mathrm{KH}}\right| c, n+1\right\rangle\left\langle c, n+1\left|\hat{H}_{\mathrm{int}}^{\mathrm{KH}}\right| b, n\right\rangle}{E_{a}+\omega-E_{c}+i \epsilon} .
$$

Here we adopt the notation for a state vector $|a, m\rangle$, where $a$ stands for a set of quantum numbers describing the helium atom and $m$ denotes a number of laser photons. In expressions (2) and (3), $a$ is the initial (ground) state of the helium atom, $b$ is the final doubly ionized atomic state, and $c$ is the set of intermediate states for which the corresponding matrix elements have nonzero values. More details about the implementation of the KH technique can be found in Ref. [10].

In the calculation using the length gauge, we have to compute only the amplitude $M_{2}$ with the operator $\hat{H}_{\mathrm{int}}^{\mathrm{L}}=\sum_{i=1}^{2} \mathbf{F} \cdot \boldsymbol{r}_{i}$, where $\boldsymbol{F}$ is the field strength.

To perform summations over all intermediate states in Eq. (3) and its analog in the length gauge), we use the discreti- zation method developed in Ref. [11] and applied to the problem of TPDI of helium in work [12]. As a set of intermediate states in the LOPT expressions, this method uses a set obtained by diagonalization of the atomic Hamiltonian in a suitably chosen basis. A variant of the discretization procedure developed in Ref. [11], which we used in our earlier work [10] for calculation of the TICS, gives the following prescription for the computation of the spectral sums in Eq. (3) we dropped the photon numbers as redundant here). We computed the amplitude

$$
M_{2}(\epsilon)=\sum_{i} \frac{\left\langle a\left|\hat{H}_{\mathrm{eff}}^{\mathrm{KH}}\right| c_{i}\right\rangle\left\langle c_{i}\left|\hat{H}_{\mathrm{eff}}^{\mathrm{KH}}\right| b\right\rangle}{E_{a}+\omega-E_{c_{i}}+i \epsilon}
$$

where $c_{i}$ is the discrete set of states resulting from the diagonalization of the atomic Hamiltonian, for several small, but finite values of $\epsilon$, and then extrapolate the results to the $\epsilon$ $=0$ limit. For too small values of $\epsilon$, the amplitudes given by Eq. (4) diverge. This divergence manifests itself when the parameter $\epsilon$ becomes comparable in magnitude to the energy spacing of the nearby states in the vicinity of a pole. Optimum values of $\epsilon$, which can be used for the reliable extrapolation to the $\epsilon=0$ limit, are generally several times larger than these energy differences [11]. For this procedure to give reliable results, the set of $\epsilon$ values used for extrapolation should be chosen so that the density of discrete states $c_{i}$ in the vicinity of the pole of the perturbation expansion is sufficiently large. We used this recipe in Ref. [10] to obtain the TICS of TPDI of helium for the photon energies below the SI threshold. It was shown in this work that amplitudes considered as functions of $\epsilon$ indeed exhibited a plateau, followed by a rapid growth, which started at values of $\epsilon$ of the order of the energy difference of the states in the vicinity of a pole. This plateau, where $M_{2}(\epsilon)$ is a slowly varying function of $\epsilon$, could be used for reliable extrapolation.

In the present case of the SI regime, the situation is more complex. As we mentioned in the Introduction, a conventional LOPT, when applied to the sequential ionization, produces divergent results. The origin of this divergence can be easily seen from Eq. (3) and is, in fact, present for any onebody operator. The divergence is due to the fact that matrix elements between a singly ionized intermediate state $1 s^{+} k p$, where one electron is left in the ground state of the $\mathrm{He}^{+}$ion and another is in a $p$-wave continuum with momentum $k$, and the final states $\Psi_{\mathbf{k}_{1} \mathbf{k}_{2}}$, are singular [9]. The appearance of this singular behavior is most transparent if the intermediate and final continuum states are represented by Coulomb waves as in Ref. [8]. The matrix elements of a one-body operator, computed with these functions, contain terms proportional to the delta functions $\delta\left(k_{1}-k\right)$ and $\delta\left(k_{2}-k\right)$. Such terms, when substituted into Eq. (3) and integrated over momenta of the intermediate states, produce the energy denominators $E_{a}+\omega$ $+V_{\mathrm{He}^{+}}-k_{1}^{2} / 2$ and $E_{a}+\omega+V_{\mathrm{He}^{+}}-k_{2}^{2} / 2$. Using the total energy conservation $E_{a}+2 \omega=k_{1}^{2} / 2+k_{2}^{2} / 2$, these denominators can be rewritten as $k_{1}^{2} / 2-\omega+V_{\mathrm{He}^{+}}$and $k_{2}^{2} / 2-\omega+V_{\mathrm{He}^{+}}$. For photon energies $\omega>V_{\mathrm{He}^{+}}$, corresponding to the SI regime, these terms produce infinite probability to observe an electron with the energy $E=\omega-V_{\mathrm{He}^{+}}$in the final state. 
As we mentioned in the Introduction, there is a way to remove this singularity by employing LOPT based on the amplitude equations taking into account depletion effects. [7]. In this approach, the depletion of the initial and intermediate states is taken into account. This implies, that in LOPT, based on the set of these amplitude equations, the energies of initial and intermediate states acquire imaginary parts proportional to the decay rates of these states. An account of these imaginary parts in the LOPT energy denominators removes the divergences, which we discussed above.

In the present work we use the conventional form of LOPT, leading to Eq. (4) with real energies. The divergence in the SDCS should be present in our approach, therefore. We shall see later that it manifests itself through the absence of the "plateau" in the calculations of the SDCS as functions of the parameter $\epsilon$. The SDCS grows monotonously with $\epsilon$, not allowing one to perform any reasonable extrapolation to the $\epsilon=0$ limit. Such behavior can be anticipated beforehand on the basis of the discussion presented in [7]. It is shown in this work that in the version of LOPT taking into account depletion of initial and intermediate states, the terms that causea divergence in the SDCS in a conventional approach assume the Lorentzian form $A /\left[\left(E-\omega+V_{\mathrm{He}^{+}}\right)^{2}+\Gamma^{2}\right)$. In this formula $E$ is the photoelectron energy, $A$ is a constant, and $\Gamma$ describes the depletion effects. If depletion effects are not included in the theory and we put $\Gamma=0$, this expression reproduces the divergent behavior of the SDCS found in [8] on the basis of conventional LOPT. From the purely formal point of view, the role of depletion effects in this respect consists in the introduction of the regularization factor $\Gamma$ in the expression for the SDCS obtained within conventional LOPT. The regularization procedure we employed above in Eq. (4) is mathematically very similar. Indeed, inclusion of finite $\epsilon$ in Eq. (4) has the same effect as prescribing finite widths to the initial and intermediate states. For finite values of $\epsilon$ we can expect, therefore, to obtain the Lorentzian profiles for the SDCS in the vicinity of the points $E_{\text {slow }}, E_{\text {fast }}$. From what has been said above, it is clear that for $\epsilon \rightarrow 0$, we should expect neither convergence nor the presence of a plateau for the SDCS as function of $\epsilon$.

The $\epsilon$ dependence of the angular anisotropy parameters, however, does show the existence of a region where these parameters are slowly (in fact, very slowly) varying functions of $\epsilon$. Possible reasons for this behavior will be discussed below. First, we give more numerical details about the basis set we used in the calculation to implement the discretization procedure and represent the initial state of helium atom.

\section{B. Atomic states}

For a reliable implementation of the discretization procedure, we have to guarantee that density of the discretized states in the vicinity of the pole in Eq. (4) is high enough. To achieve this goal, we used the basis constructed from a set of $B$ splines in a box, which we employed previously for calculations of the TICS in the NSI regime [10].

In the present calculation, we used a larger box size, which resulted in a slight modification of the $B$ spline set.
Details of this set are given below. We use a set of $B$ splines of the order $k=7$ with the knots located at the sequence of points lying in $\left[0, R_{\max }\right]$. The bulk of the results presented below have been obtained for $R_{\max }=130$ a.u. To demonstrate stability of convergence of the results with the box size, we also performed a separate calculation for $R_{\max }=100$ a.u.

A typical number of knot points was 40-45. All the knots $t_{i}$ were simple ones, except for knots located at the origin and the outer boundary $R=R_{\max }$ of the box. These knots had multiplicity $k=7$. The simple knots were distributed in $\left(0, R_{\max }\right)$ according to the rule $t_{i+1}=\alpha t_{i}+\beta$, with $\beta=0.65$ and $\alpha$ close to 1 (typically 1.1 ).

For each value of the angular momentum $l$, the first $l+1 B$ splines and the last $B$ spline resulting from this sequence of knots were discarded. Omission of the first $l+1 B$ splines ensured that any $B$ spline in the set decreased as $r^{l+1}$ (or faster) at the origin; omission of the last $B$ spline ensured that all $B$ splines of a set assumed zero value at the outer boundary. The set of $B$ splines constructed for each $0 \leqslant l \leqslant 3$ served as a set of one-electron radial functions $R_{l}(r)$. The twoelectron basis functions were built from these orbitals in the usual way. For the calculation using the box size of $R_{\max }$ $=130$ a.u., we had 2180 basis functions of $S$ symmetry and 3170 basis functions of $P$ symmetry. The former were used for the representation of the ground state of the helium atom. Diagonalization of the helium Hamiltonian in the subspace of the basis functions of $S$ symmetry gave the ground-state energy $E_{a}=-2.90273$ a.u. The basis functions of $P$ symmetry served for the construction of the set of the intermediate states $c_{i}$ in Eq. (4). In the vicinity of the pole of the LOPT expansion (4), the energy spacing of the discretized states was typically several thousandth of an a.u., providing a sufficiently dense representation of the continuum of $P$ states.

When checking the stability of the results with the box size, we used $R_{\max }=100$ a.u. Using a knot distribution similar to the one described above for the $R_{\max }=130$ a.u. calculation, we obtained 2050 basis functions of $S$ symmetry and 2978 basis functions of $P$ symmetry.

The final states, which we needed for TPDI calculations, were states of $S$ and $D$ symmetry with incoming boundary conditions describing two electrons in continuum. We used the convergent close-coupling (CCC) method [13] to represent these states. In this method, the two-electron scattering state is represented by a close-coupling expansion over the channel states composed of a target pseudostate $f$ and a Coulomb wave $\boldsymbol{k}$ :

$$
\Psi_{f}(\boldsymbol{k})=|\boldsymbol{k} f\rangle+\sum_{\boldsymbol{k}^{\prime} j} \frac{\left\langle\boldsymbol{k} f|T| \boldsymbol{k}^{\prime} j\right\rangle}{E-k^{\prime 2} / 2-\varepsilon_{j}-i 0}\left|\boldsymbol{k}^{\prime} j\right\rangle
$$

Here $\left\langle\boldsymbol{k} f|T| \boldsymbol{k}^{\prime} j\right\rangle$ is the half-on-shell $T$ matrix, which is found by solving a set of coupled Lippmann-Schwinger integral equations [14]. For the target pseudostates $f$ with positive energy $\varepsilon_{f}>0$, the wave function given by Eq. (5) can be used to construct a state with two electrons in continuum [13]: 


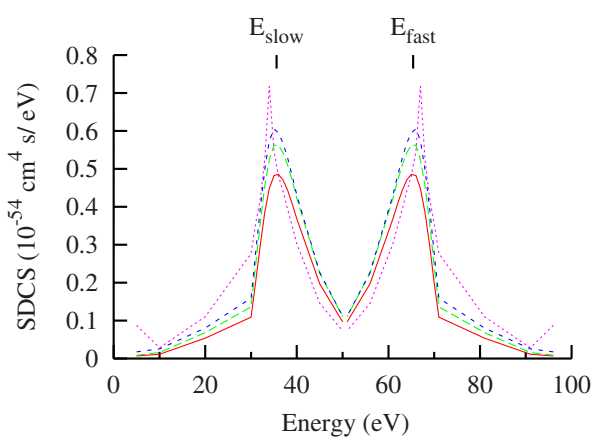

FIG. 1. (Color online) Single differential cross section at the photon energy of $90 \mathrm{eV}$ and different values of the regularization parameter: $\epsilon=0.1$, solid (red) line; $\epsilon=0.05$, long dashed (green) line; $\epsilon=0.01$, dashed (blue) line. The photoelectron energies in the absence of correlation, $E_{\text {slow }}=35.6 \mathrm{eV}$ and $E_{\text {fast }}=65.4 \mathrm{eV}$, are indicated by the tic marks.

$$
\begin{aligned}
\Psi^{-}\left(\boldsymbol{k}_{1}, \boldsymbol{k}_{2}\right)= & \sum_{J=0,2} \sum_{l_{1} l_{f}} \mathcal{Y}_{J 0}^{l_{1} l_{f}}\left(\hat{\boldsymbol{k}}_{1}, \hat{\boldsymbol{k}}_{2}\right) i^{l_{1}+l_{f}} e^{-i\left[\delta_{l_{1}}^{Z=1}\left(k_{1}\right)+\delta_{l_{f}}^{Z=2}\left(k_{2}\right)\right]} \\
& \times\left\langle n_{f} l_{f} \mid k_{2}, l_{2}\right\rangle \Psi_{n_{f} l_{f} l_{1} ; j}\left(k_{1}\right) .
\end{aligned}
$$

In Eq. (6) we introduced a bipolar harmonic according to Ref. [15]:

$$
\mathcal{Y}_{J M}^{l_{1} l_{2}}\left(\hat{\boldsymbol{k}}_{1}, \hat{\boldsymbol{k}}_{2}\right)=\sum_{m_{1} m_{2}} C_{l_{1} m_{2}, l_{2} m_{2}}^{J M} Y_{l_{1} m_{1}}\left(\hat{\boldsymbol{k}}_{1}\right) Y_{l_{2} m_{2}}\left(\hat{\boldsymbol{k}}_{2}\right) .
$$

The unit vectors $\hat{\boldsymbol{k}}_{i}=\boldsymbol{k}_{i} / k_{i}$ are directed along the photoelec-

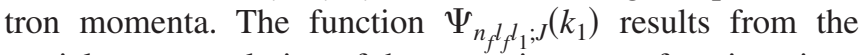
partial-wave resolution of the scattering wave function given by Eq. (5); $\left\langle n_{f} l_{f} \| k_{2}, l_{2}\right\rangle$ is a radial overlap of the target pseudostate and a Coulomb wave with $Z=2$ and matching energy $k_{2}^{2} / 2=\epsilon_{f}$. Finally, $\delta_{l}^{Z=1}\left(k_{1}\right)$ and $\delta_{l_{f}}^{Z=2}\left(k_{2}\right)$ are the Coulomb phases with corresponding nucleus charges. More details on the implementation of this procedure can be found in Refs. $[13,16]$.

\section{Calculation of SDCS, TDCS, and angular anisotropy parameters}

By plugging the atomic states described in the previous section into amplitudes of Eqs. (2) and (3), we can compute the TDCS of a process in which absorption of two photons leaves the helium atom in a state with two electrons in continuum with momenta $\boldsymbol{k}_{1}$ and $\boldsymbol{k}_{2}$. With normalization of the continuum wave functions to the $\delta$ function of momentum, this cross section (in units of $\mathrm{cm}^{4} \mathrm{~s} / \mathrm{eV}$ ) is given by the following expression:

$$
\frac{d \sigma}{d E d \Omega_{1} d \Omega_{2}}=\frac{2^{7} \pi^{3} \alpha^{2} a_{0}^{4} \tau \omega^{2}}{\mathcal{R}} \frac{\left|M_{1}\left(\boldsymbol{k}_{1}, \boldsymbol{k}_{2}\right)+M_{2}\left(\boldsymbol{k}_{1}, \boldsymbol{k}_{2}\right)\right|^{2}}{F^{4} k_{1} k_{2}} .
$$

Here $\alpha$ is the fine-structure constant, $a_{0}=0.529 \times 10^{-8} \mathrm{~cm}$ is the Bohr radius, $\tau=2.418 \times 10^{-17} \mathrm{~s}$ is the atomic unit of time, $\mathrm{R}=27.211 \mathrm{eV}$ is the Rydberg constant, and $k_{1}$ and $k_{2}$ are photoelectron momenta. The notation $M\left(\boldsymbol{k}_{1}, \boldsymbol{k}_{2}\right)$ is used for the amplitudes in Eqs. (2) and (3), computed with the final- state wave function given by Eq. (6). The SDCS is obtained by integration of Eq. (8) over the solid angles $d \Omega_{1} d \Omega_{2}$. Finally, the angular anisotropy parameters $\beta_{2}$ and $\beta_{4}$ can be found by integration of Eq. (8) over one of the solid angles and representing the result as a Legendre polynomial expansion:

$$
\frac{d \sigma}{d E d \Omega}=\frac{d \sigma}{d E} \frac{1}{4 \pi}\left[1+\beta_{2} P_{2}(\cos (\theta))+\beta_{4} P_{4}(\cos (\theta))\right] .
$$

As we discussed above, the cross section (8) is divergent in the present LOPT approach. Consequently, the cross sections $d \sigma /(d E d \Omega)$ and $d \sigma / d E$ in Eq. (9) are divergent. However, their ratio, which determines the $\beta$ parameters, is not. It is due to this reason that LOPT, in its present version, is capable of providing meaningful values for the $\beta$ parameters, as we shall try to demonstrate below.

\section{RESULTS}

In Fig. 1 we show our results for the SDCS calculated at the photon energy of $90 \mathrm{eV}$. The $\mathrm{KH}$ gauge calculations with the box size $R_{\max }=130$ a.u. are displayed for different values of the regularization parameter $\epsilon$. At this photon energy, the separation of the discretized states in Eq. (4) in the vicinity of the pole was approximately 0.003 a.u. If any sort of convergence of the SDCS with respect to $\epsilon$ could be achieved, we should expect it to be visible on the plot. The value of $\epsilon=0.01$ lies, perhaps, dangerously close to the critical value as determined by the energy differences in the vicinity of the pole. Therefore, the discretization procedure is likely to fail in this case. We do not see, however, convergence for larger values of $\epsilon$ either. The SDCSs calculated with $\epsilon=0.1$ and $\epsilon=0.05$ vary considerably. Instead of showing a "plateau" for values of $\epsilon$ several time larger than the critical value, the SDCS exhibits steady growth, not allowing for meaningful extrapolation to the $\epsilon=0$ limit.

Now we turn our attention to the asymmetry $\beta$ parameters. First, we perform several checks to show that our results do not depend on various auxiliary parameters, characterizing the calculation.

We show results of these checks in Fig. 2, where we display the anisotropy $\beta_{2}$ and $\beta_{4}$ parameters at the photon energy of $90 \mathrm{eV}$. For the reference calculation, we choose the $\mathrm{KH}$ gauge, the box size $R_{\max }=130$ a.u., and the regularization parameter $\epsilon=0.1$. In the left panel, we reduce the box size to $R_{\max }=100$ a.u., which has almost no visible effect on the $\beta$ parameters. This check assures us of the stability of our calculation with respect to the box size, which otherwise could have suffered from the appearance of spurious unphysical resonances [17].

A small discontinuity of the $\beta$ parameters at the midpoint is an inherent feature of the CCC method, which treats the two photoelectrons on different footing. As is seen from Eq. (6), one-usually the slow photoelectron-is described by the target pseudostates, while another-the fast photoelectron-is described by the Coulomb waves. Direct calculation of the TDCS and $\beta$ parameters is only possible for $E_{2}<E_{1}$. Another half of the photoelectron energy interval is covered by the assumed symmetry of the wave function 

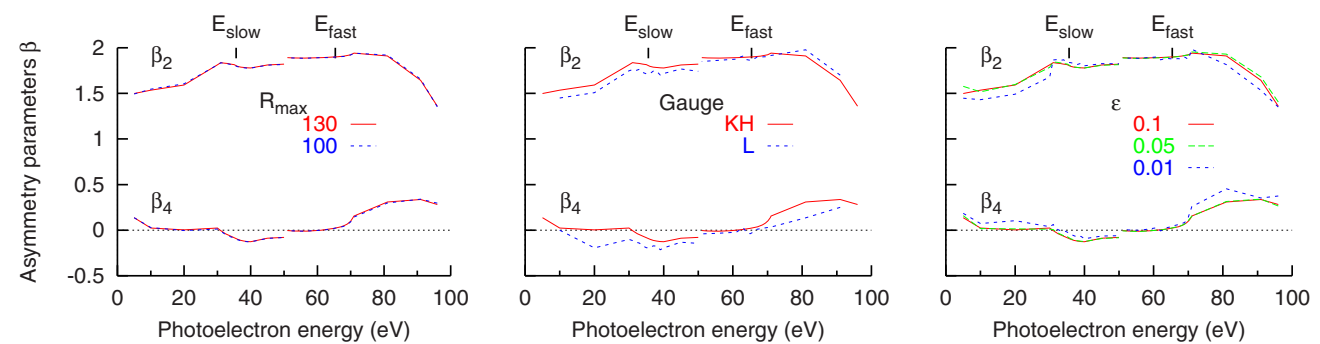

FIG. 2. (Color online) Photoelectron asymmetry parameters $\beta_{2}$ (top) and $\beta_{4}$ (bottom) for the photon energy of $90 \mathrm{eV}$. The reference KH gauge calculation with the box size $R_{\max }=130$ a.u. and $\epsilon=0.1$ is plotted on all panels with the solid (red) line. Left panel: the box size is changed to $R_{\max }=100$ a.u., dashed (blue) line. Middle panel: the KH is changed to the length gauge, dashed (blue) line. Right panel: the $\epsilon$ parameter is changed to $\epsilon=0.05$, long dashed (green) line, and $\epsilon=0.01$, dashed (blue) line.

(6) with respect to exchange of electron momenta $\boldsymbol{k}_{1} \leftrightarrow \boldsymbol{k}_{2}$. In a numerical calculation, this symmetry is approximate and holds only to a certain degree. This effect is well known [18] and manifests itself only in the energy region very close to the midpoint. Unlike the $\beta$ parameters, the SDCS plotted in Fig. 1 is symmetrized with respect to the midenergy point and shows no visible gap.

As another check of the consistency of the present calculation, we compare the reference set of $\beta$ parameters with an analogous calculation in the length gauge (middle panel of Fig. 2). The two gauges agree reasonably well. Strictly speaking, for finite values of $\epsilon$, gauge invariance need not hold [11]; it is restored only for the values extrapolated to the $\epsilon=0$ limit. We believe that, as in our previous calculation of TICSs below the SI threshold [10], the KH gauge results are considerably more accurate. As one can see, the length gauge results show some rather unphysical oscillations. A convenient feature of the $\mathrm{KH}$ gauge is the fast decrease of the electromagnetic operator with distance. The $\mathrm{KH}$ gauge thus emphasizes the regions of the box close to the nucleus, where, as our rather accurate ground state energy value indicates, we may hope to have achieved a good description of the electronic structure of the target. That also explains why our results are so insensitive to the box size. The length gauge, on the contrary, puts emphasis on the outer regions of the box and may suffer from spurious unphysical effects to a greater degree.

Finally, in the right panel of Fig. 2, we compare the reference calculation with the ones in which the regularization $\epsilon$ parameter varies as in Fig. 1. As one can see, for $\epsilon=0.1$ and $\epsilon=0.05$, the $\beta$ parameters are virtually identical in the whole range of the photoelectron energies. Even for the value $\epsilon$ $=0.01$, which, as we determined above, is perhaps too close to the critical value, deviation of the $\beta$ values is not that large. This, we believe, is a consequence of the fact that determination of the $\beta$ parameters does not rely on the SDCS, which diverges in the version of LOPT that we employ.

It is worthwhile to compare our $\beta$ parameters with those which follow from a simple model of TPDI in the SI regime proposed in Ref. [8]. This model neglects correlations in the intermediate and final states and represents TDCSs as a product of two dipole distributions for each of the photoelectrons. Consequently, in this model $\beta_{2}=2$ and $\beta_{4}=0$ in the whole photoelectron energy range. This prediction agrees reasonably well with our data at not too extreme energy sharings, where deviation from the dipole regime becomes most noticeable. The authors of Ref. [2] observed themselves that their simple model cannot provide an accurate description of the photoelectron angular distribution for all possible electron energy sharings. Indeed, they demonstrated that for a photon energy of $58 \mathrm{eV}$ and $90 \%$ of the excess energy carried by the fast photoelectron, the TDCS differs dramatically from the dipole distribution. Present results apparently confirm this statement.

For a photon energy of $90 \mathrm{eV}$, in the absence of correlation, the energies of the photoelectrons should be equal to $E_{\text {slow }}=35.6 \mathrm{eV}$ and $E_{\text {fast }}=65.4 \mathrm{eV}$. These values are marked by corresponding tic marks in the top horizontal scale of Fig. 2 . As one can see, in between these energies, $\beta_{2}$ is indeed close to 2 , while $\beta_{4}$ is relatively small. We observe a significant departure from this simple dipole regime only in the case of extreme energy sharings, either for $E<E_{\text {slow }}$ or for $E>E_{\text {fast }}$.

This statement can, perhaps, be raised to the status of a propensity rule. We illustrate this rule in Fig. 3, where we present plots for $\beta$ parameters for photon energies of 62.7, 90 , and $150 \mathrm{eV}$. These results have been obtained with a box
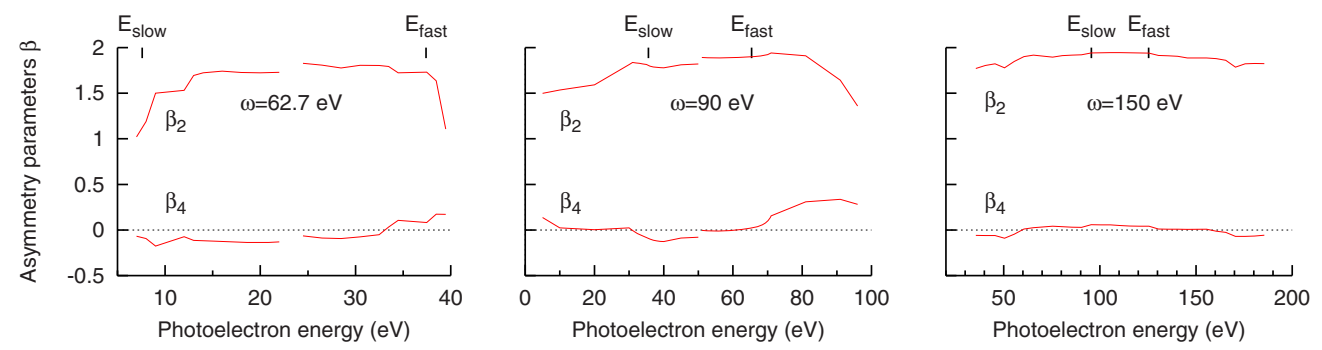

FIG. 3. (Color online) $\beta$ parameters for photon energies of $62.7 \mathrm{eV}, 90 \mathrm{eV}$, and $150 \mathrm{eV}$. 
size of 130 a.u., $\epsilon=0.1$, and the KH gauge. We have performed the same set of checks for all photon energies as described above for $90 \mathrm{eV}$ in order to ensure that neither the box size nor the choice of the regularization $\epsilon$ parameter or gauge affects significantly our numerical results. For the reader's convenience, we put in the middle panel of Fig. 3 the reference data set for $90 \mathrm{eV}$ photon energy, which we presented already in Fig. 2.

As one can see from Fig. 3, $\beta_{2}$ remains close to 2 for electron energie in the interval $\left(E_{\text {slow }}, E_{\text {fast }}\right)$. For electron energies outside this interval, $\beta_{2}$ deviates from 2 , this deviation becoming less pronounced with an increase of the photon energy.

One may give an explanation of such a behavior of the parameter $\beta_{2}$ using the following simple picture. Let us consider TPDI in the SI regime as a sequence of two ionization events. To produce photoelectrons with energies outside the interval $\left(E_{\text {slow }}, E_{\text {fast }}\right)$, the following has to happen. The fast electron, emerging first with energy $E_{\text {fast }}$, has to accelerate further yet. If we neglect electron correlation completely (as in a simple model employed in Ref. [8]) or even describe this correlation within a simplified static exchange model, then the fast electron will see the overall attractive potential of the $\mathrm{He}^{+}$ion. This attractive potential can only decelerate the electron. For the fast electron to acquire additional energy, correlations must play a major role. On the contrary, if electron energies in the final state lie in the interval $\left(E_{\text {slow }}, E_{\text {fast }}\right)$, the fast electron has to decelerate. This is a process which can occur without any correlations at all, the attractive potential of the $\mathrm{He}^{+}$ion being enough to produce such an effect. This may explain the behavior of the $\beta_{2}$ parameter seen in Fig. 2 and the fact that in the interval $\left(E_{\text {slow }}, E_{\text {fast }}\right)$ this behavior does not differ considerably from the simple dipole behavior.

As to the parameter $\beta_{4}$, this is a more subtle characteristic. This parameter is determined by correlations in all accessible interval of energies. Any model for $\beta_{4}$ should, therefore, necessarily include correlations, which makes it difficult to provide an explanation for the observed behavior of $\beta_{4}$ in simple terms.

Generally, the deviation of $\beta_{2}$ from 2 and $\beta_{4}$ from 0 becomes less prominent as the photon energy grows. This is quite natural, as electron correlation plays a less important role in the emission of fast photoelectrons, which leave the atom quickly and have no time to interact with each other.

\section{CONCLUSION}

In the present work, we studied photoelectron angular distributions following the process of two-photon double electron ionization of $\mathrm{He}$ in the sequential regime at the photon energies of $62.7,90$, and $150 \mathrm{eV}$. Our study was motivated by the previous works on TPDI of He in the SI regime $[5,6]$. These works demonstrated that, for a laser pulse duration of several optical cycles, electron correlation leaves its trace in the so-called "correlation valley," the region close to the midenergy point in SDCSs, where an anomalous component of the SI is present. It was also shown in Ref. [6] that the correlation valley displays an anomalous behavior as far as the angular distribution of photoelectrons is concerned. The corresponding angular anisotropy $\beta$ parameters assume values very different from the simple dipolar behavior one would expect in the absence of electron correlation.

For long laser pulses, the correlation valley should disappear. Our results for $\beta$ parameters indicate that, indeed, in the whole region of energies $\left(E_{\text {slow }}, E_{\text {fast }}\right)$ the angular distribution is of a simple dipole character. This is the result one would expect for the uncorrelated electrons. This behavior is in marked contrast with the behavior of the $\beta$ parameters for TPDI at photon energies below the SI threshold. For a photon energy of $45 \mathrm{eV}$ [18], for example, $1<\beta_{2}<1.4$, thus indicating an important role played by correlations for all accessible electron energies.

The question which we posed to ourselves was if there were other traces of correlation left for sufficiently long pulses. Such traces are indeed present for extreme energy sharings of photoelectron outside the interval $\left(E_{\text {slow }}, E_{\text {fast }}\right)$. In this energy region, we found a noticeable deviation of $\beta_{2}$ from 2 and $\beta_{4}$ from zero. This region is only accessible due to electron correlation, which upsets the energy balance between the photoelectrons. An overall correlation effect is more pronounced for smaller photon energy, where correlation plays a more prominent role.

\section{ACKNOWLEDGMENTS}

The authors acknowledge support of the Australian Research Council in the form of the Discovery Grant No. DP0771312. Resources of the National Computational Infrastructure (NCI) Facility were used.
[1] P. Lambropoulos, L. A. A. Nikolopoulos, and M. G. Makris, Phys. Rev. A 72, 013410 (2005).

[2] D. A. Horner, T. N. Rescigno, and C. W. McCurdy, Phys. Rev. A 77, 030703(R) (2008).

[3] J. S. Parker, L. R. Moore, K. H. Meharg, D. Dundas, and K. T. Taylor, J. Phys. B 34, L69 (2001).

[4] S. Laulan and H. Bachau, Phys. Rev. A 68, 013409 (2003).

[5] K. L. Ishikawa and K. Midorikawa, Phys. Rev. A 72, 013407 (2005).

[6] I. F. Barna, J. Wang, and J. Burgdorfer, Phys. Rev. A 73,
023402 (2006).

[7] P. Lambropoulos, L. A. A. Nikolopoulos, M. G. Makris, and A. Mihelič, Phys. Rev. A 78, 055402 (2008).

[8] D. A. Horner, F. Morales, T. N. Rescigno, F. Martin, and C. W. McCurdy, Phys. Rev. A 76, 030701(R) (2007).

[9] D. Proulx, M. Pont, and R. Shakeshaft, Phys. Rev. A 49, 1208 (1994).

[10] I. A. Ivanov and A. S. Kheifets, J. Phys. B 41, 095002 (2008).

[11] E. Cormier and P. Lambropoulos, J. Phys. B 28, 5043 (1995).

[12] L. A. A. Nikolopoulos and P. Lambropoulos, J. Phys. B 34, 
545 (2001).

[13] I. Bray, Phys. Rev. A 49, 1066 (1994).

[14] I. Bray and A. T. Stelbovics, Adv. At., Mol., Opt. Phys. 35, 209 (1995).

[15] D. A. Varshalovich, A. N. Moskalev, and V. K. Khersonskii, Quantum Theory of Angular Momentum (World Scientific,
Singapore, 1988).

[16] A. S. Kheifets and I. Bray, J. Phys. B 31, L447 (1998).

[17] P. G. Burke, K. A. Berrington, and C. V. Sukumar, J. Phys. B 14, 289 (1981).

[18] A. S. Kheifets, I. A. Ivanov, and I. Bray, Phys. Rev. A 76, 025402 (2007). 